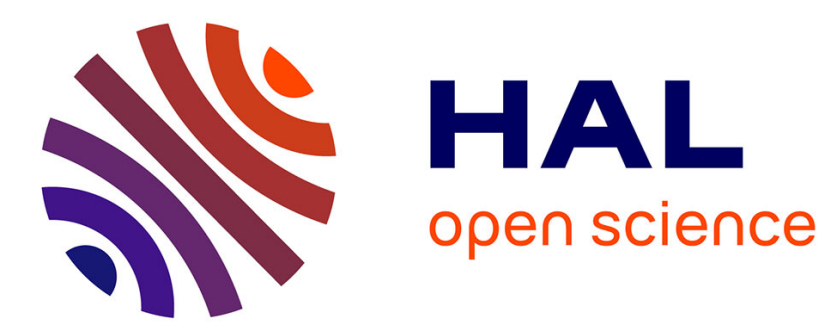

\title{
Smallest focal spots
}

T. Grosjean, D. Courjon

\section{To cite this version:}

T. Grosjean, D. Courjon. Smallest focal spots. Optics Communications, 2006, 272 (2), pp.314-319. 10.1016/j.optcom.2006.11.043 . hal-00215997

\section{HAL Id: hal-00215997 \\ https://hal.science/hal-00215997}

Submitted on 13 May 2021

HAL is a multi-disciplinary open access archive for the deposit and dissemination of scientific research documents, whether they are published or not. The documents may come from teaching and research institutions in France or abroad, or from public or private research centers.
L'archive ouverte pluridisciplinaire HAL, est destinée au dépôt et à la diffusion de documents scientifiques de niveau recherche, publiés ou non, émanant des établissements d'enseignement et de recherche français ou étrangers, des laboratoires publics ou privés. 


\title{
Smallest focal spots
}

\author{
T. Grosjean *, D. Courjon \\ Département d'Optique P.M. Duffieux, Institut FEMTO-ST, UMR CNRS 6174, Université de Franche-Comté, \\ 16 route de Gray, 25030 Besanc, on cedex, France
}

It is commonly admitted that radial polarization leads to the best light beam focalisation in terms of spot size. The work presented here shows that the choice of polarization is in fact more subtle. As an example, it is pointed out that, for numerical apertures of focusing systems below 0.8 , circular polarization induces the best light confinements in air, whereas for numerical apertures beyond 0.8 , radial polarization seems to be the right solution. Moreover, for numerical apertures larger than 1, we propose an original solution to achieve the ultimate focal spots in linear optical regime. They are estimated to $0.36 \lambda / \mathrm{NA}$ ( $\lambda$ is the wavelength and NA is the numerical aperture), the current technological limit being around $65 \mathrm{~nm}$.

Keywords: Radial polarization; Circular polarization; Bessel beams; Conical optics; High index of refraction; Superresolution; Focusing optimization; Nano-optics; Near field optics; Virtual tip

\section{Introduction}

In previous papers [1,2], Bessel beams have been presented as one powerful solution to generate confined focal spots usable in superresolution imaging [3-9]. Ref. [10] points out that polarization plays a crucial role in the definition of the light spot of Bessel beams. For example, it has been numerically demonstrated that the spot size of evanescent Bessel beams is optimized in radial polarization. This conclusion has been previously drawn for focused beams [11]. In this paper, we will go further in the search of highest confinements through a complete analysis of the effect of polarization on the light spots generated by Bessel beams. The goal is first to explain the effect of polarization on the limitation of the confinement lateral extension, and second to study which polarization induces in air the smallest focal spots, whatever the numerical aper-

\footnotetext{
${ }^{*}$ Corresponding author. Tel.: +33 (0)3 816664 15; fax: +33 (0)3 8166 6423.

E-mail address: thierry.grosjean@univ-fcomte.fr (T. Grosjean).
}

ture (NA) of the focusing device. Finally, we will propose the first solution to our knowledge to achieve the ultimate focal spots. It will be shown that such a situation requires the master of NA, polarization and the index of refraction of the medium where the Bessel beam is created.

\section{Light confining process of Bessel beams, the role of polarization}

Let us consider the system which consists of two semiinfinite media limited by a plane interface (see Fig. 1). A Bessel beam propagates in the first dielectric medium (of refraction index $n$ ). It is characterized by an aperture angle $\theta$ defined by the relation NA $=n \sin \theta$. Depending on NA, the transmitted field describes either a propagative Bessel beam $(\mathrm{NA}<1)$ or an evanescent one (NA $>1)$. The transmission coefficients associated to the plane interface provide a simple model of the immersion systems allowing the generation of propagative or evanescent Bessel beams $[12,13]$. 


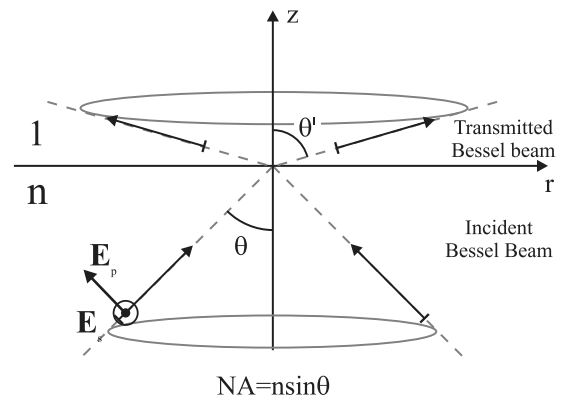

Fig. 1. Scheme of the configuration considered in this study.

Theoretical study of focused beams [2,14-18] and Bessel beams $[10,19-21]$ in high-NA regime points out that the spatial shape of the focal spot strongly depends on the incident-field polarization. It turns out that rotationally symmetric light spots are generated with incident beams exhibiting either isotropic or rotationally symmetric polarizations. Among the commonly used polarizations, only isotropic circular polarization and rotationally symmetric radial and azimuthal polarizations provide circular spots. Because the generation of rotationally symmetric light spots is highly desirable for many applications such as confocal microscopy, lithography and data storage (it involves isotropic optical properties with respect to the scan directions), we will limit our analysis to those three polarization states.

From Refs. [22,2,16-18], analytical expressions of the field distributions describing the transmitted Bessel beams can be written in circular polarization as

$$
\begin{aligned}
E_{x} \propto & \exp [\mathrm{i} w z]\left[\left(t_{\mathrm{s}}+t_{\mathrm{p}_{r}} \cos \theta\right) J_{0}(\alpha r)\right. \\
& \left.+\left(t_{\mathrm{s}}-t_{\mathrm{p}_{r}} \cos \theta\right) \exp [\mathrm{i} 2 \xi] J_{2}(\alpha r)\right], \\
E_{y} \propto & \mathrm{i} \exp [\mathrm{i} w z]\left[\left(t_{\mathrm{s}}+t_{\mathrm{p}_{r}} \cos \theta\right) J_{0}(\alpha r)\right. \\
& \left.-\left(t_{\mathrm{s}}-t_{\mathrm{p}_{r}} \cos \theta\right) \exp [\mathrm{i} 2 \xi] J_{2}(\alpha r)\right], \\
E_{z} \propto & -2 \mathrm{i} \exp [\mathrm{i} w z] \sin \theta t_{\mathrm{p}_{z}} \exp [\mathrm{i} \xi] J_{1}(\alpha r),
\end{aligned}
$$

in radial polarization as

$E_{x} \propto-\mathrm{i} \exp [\mathrm{i} w z] t_{\mathrm{p}_{r}} \cos \theta \cos \xi J_{1}(\alpha r)$,

$E_{y} \propto-\mathrm{i} \exp [\mathrm{i} w z] t_{\mathrm{p}_{r}} \cos \theta \sin \xi J_{1}(\alpha r)$,

$E_{z} \propto \exp [\mathrm{i} w z] t_{\mathrm{p}_{z}} \sin \theta J_{0}(\alpha r)$,

and in azimuthal polarization as

$E_{x} \propto \mathrm{i} \exp [\mathrm{i} w z] t_{\mathrm{s}} \sin \xi J_{1}(\alpha r)$,

$E_{y} \propto-\mathrm{i} \exp [\mathrm{i} w z] t_{\mathrm{s}} \cos \xi J_{1}(\alpha r)$,

$E_{z}=0$.

Parameters $r, \xi$ and $z$ are cylindrical spatial-coordinates, $\alpha$ is a constant and $J_{m}(\alpha r)$ are the $m$-order Bessel functions of the first kind. Coefficient $w$ is defined as $w=\sqrt{\left(k^{2}-\alpha^{2}\right)}$ where $k$ is the wave number. $t_{\mathrm{p}_{r}}, t_{\mathrm{p}_{z}}, t_{\mathrm{s}}$ are the transmission coefficients of the $\mathrm{p}$ and $\mathrm{s}$ components of the vectorial angular spectrum defining the transmitted Bessel beam.
The intensity expressions $I_{\text {circ }}, I_{\text {rad }}$ and $I_{\text {azi }}$ in circular, radial and azimuthal polarizations, respectively, can be derived from Eqs. (1)-(9) by calculating the field square modulus. We find:

$I_{\text {circ }}(\vec{r}) \propto J_{0}^{2}(\alpha r)+K_{2}^{\text {circ }} J_{2}^{2}(\alpha r)+K_{1}^{\text {circ }} J_{1}^{2}(\alpha r)$,

$I_{\mathrm{rad}}(\vec{r}) \propto J_{0}^{2}(\alpha r)+K_{1}^{\mathrm{rad}} J_{1}^{2}(\alpha r)$,

$I_{\mathrm{azi}}(\vec{r}) \propto\left|t_{\mathrm{s}}\right|^{2} J_{1}^{2}(\alpha r)$.

Eqs. (10)-(12) can be seen as the combinations of square Bessel functions whose orders and weighting coefficients $K_{1}^{\text {circ }}, K_{2}^{\text {circ }}, K_{1}^{\text {rad }}$ and $\left|t_{\mathrm{s}}\right|^{2}$ are defined by polarization.

We have:

$$
\begin{aligned}
K_{1}^{\text {circ }} & =\frac{2\left|t_{\mathrm{p}_{z}} \sin \theta\right|^{2}}{\left|t_{\mathrm{s}}+t_{\mathrm{p}_{r}} \cos \theta\right|^{2}}, \\
K_{2}^{\text {circ }} & =\frac{\left|t_{\mathrm{s}}-t_{\mathrm{p}_{r}} \cos \theta\right|^{2}}{\left|t_{\mathrm{s}}+t_{\mathrm{p}_{r}} \cos \theta\right|^{2}}, \\
K_{1}^{\mathrm{rad}} & =\frac{\left|t_{\mathrm{p}_{r}} \cos \theta\right|^{2}}{\left|t_{\mathrm{p}_{z}} \sin \theta\right|^{2}}, \\
& =\left|1-\frac{1}{\mathrm{NA}}\right|,
\end{aligned}
$$

Eq. (16) is deduced from Eq. (15) by replacing $t_{\mathrm{p}_{r}}$ and $t_{\mathrm{p}_{z}}$ by their expressions as functions of NA.

In Fig. 2 are depicted the square Bessel functions involved in the intensity expressions of Eqs. (10)-(12) It turns out that only $J_{0}^{2}$ exhibits a non null value at the origin. Since the generation of a light spot requires a non null central intensity, Bessel beams (and more generally, focused beams) can generate a light spot provided that the polarization produces function $J_{0}$ in one of the three field components at least. In other words, if $J_{0}^{2}$ is not in the intensity expression, the Bessel beam exhibits a dark central spot, whatever NA. Thus, we see from Eq. (12) that no light confinement can be generated with azimuthally polarized Bessel beams. Such beams give the smallest dark spot which can be generated by focusing systems [23,2].



Fig. 2. Plots of functions $J_{0}^{2}, J_{1}^{2}$ and $J_{2}^{2}$. 
This case will not be discussed here. On the other hand, circularly and radially polarized Bessel beams can exhibit a central light spot.

From Fig. 2, we see that the central lobe of $J_{0}^{2}$ defines the ultimate light spot which can be generated by Bessel beams, therefore by focusing. According to the location of their respective first maxima, functions $J_{1}^{2}$ and $J_{2}^{2}$ tend to enlarge more or less this primary light confinement, depending on their weighting coefficients. We conclude that the influence of polarization on the spot size is fully controlled by the weighting coefficients of functions $J_{1}^{2}$ and $J_{2}^{2}$, i.e., coefficients $K_{1}^{\text {circ }}, K_{2}^{\text {circ }}$ in circular polarization and $K_{1}^{\text {rad }}$ in radial polarization.

Then, the role of polarization on the light confinement process is double. First, it fully defines whether a light spot can be generated or not. Second, it participates in the extension of the light spot by setting the relative weights of the various Bessel functions involved in the intensity expression. In the next section, the influence of polarization on the spot size we will be analyzed.

\section{Polarization effects in the spot size limitation}

Figs. 3 and 4 display coefficients $K_{1}^{\text {circ }}, K_{2}^{\text {circ }}$ and $K_{1}^{\text {rad }}$ as functions of NA, for three different values of $n$. We note that all the curves plotted for $n>1$ exhibit a discontinuity at critical angle $\theta_{\mathrm{c}}=\arcsin (1 / n)(\mathrm{NA}=1)$ which defines the limit between the propagative and evanescent regimes for the transmitted Bessel beam. This phenomenon is due to the fact that the linearly polarized p-component $\left(E_{\mathrm{p}}\right)$ of the transmitted angular spectrum in propagative regime ( $w$ is real) become elliptically polarized in evanescent regime ( $w$ is imaginary). This field re-orientation induces strong modifications in the relative weights of the square Bessel functions.

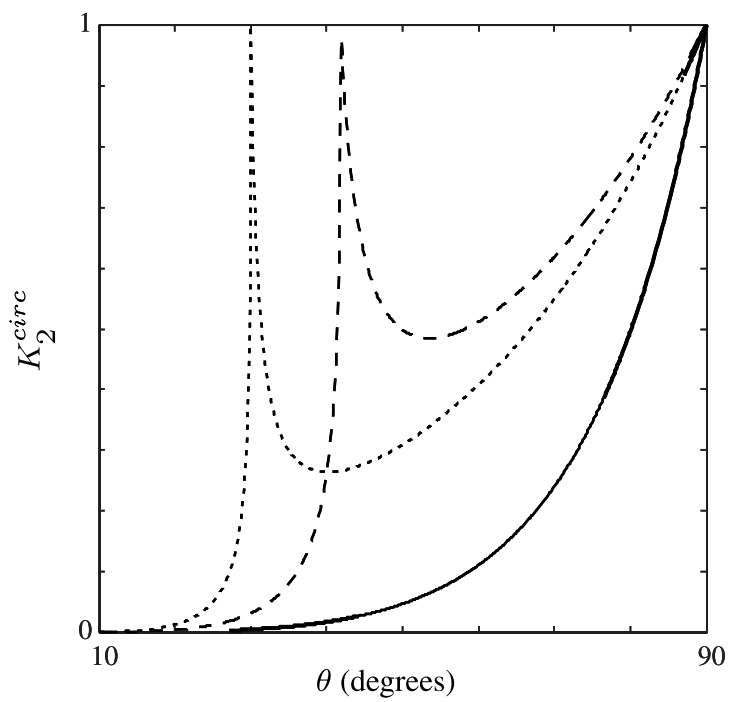

Fig. 3. Plots of coefficient $K_{2}^{\text {circ }}$ as a function of $\theta$ for $n=1$ (solid curve), $n=1.5$ (dashed curve) and $n=2$ (dotted curve).
Fig. 3 shows that $K_{2}^{\text {circ }}$ does not exceed unity whatever $n$ and $\theta$. According to the characteristics of $J_{2}^{2}$ (see Fig. 2), we can conclude that the influence of $J_{2}^{2}$ on the spot size is sufficiently weak to be neglected. The effects of circular and radial polarizations on the spot size can be fully described and quantified through coefficients $K_{1}^{\text {circ }}$ and $K_{1}^{\mathrm{rad}}$, respectively.

We conclude that the focusing optimization consists of choosing the polarization state which minimizes the weighting coefficient of function $J_{1}^{2}$ in the Bessel beam intensity expression. Fig. 4 shows that $K_{1}^{\text {circ }}<K_{1}^{\text {rad }}$ when NA $<0.8$ and $K_{1}^{\text {circ }}>K_{1}^{\text {rad }}$ otherwise, whatever $n$. Therefore, the smallest light confinements generated by focusing in air are carried by circularly polarized Bessel beams when $\mathrm{NA}<0.8$ and radially polarized Bessel beams when NA $>0.8$, whatever $n$.

To illustrate these conclusions, Fig. 5a and b display the light spots generated by circularly and radially polarized Bessel beams, respectively, when $\mathrm{NA}=0.65 \quad(n=1)$. Focusing devices of NA $=0.65$ are for example widely used in data storage (Digital Versatile Disc (DVD) and HighDefinition DVD) [24]. The Full-Width at Half-Maximum (FWHM) of the intensity profiles shown in Fig. 5c allows the estimate of the confinement lateral size. The spot size in circular polarization $(\mathrm{FWHM}=0.6 \lambda)$ is 1.64 smaller than the one obtained in radial polarization $(\mathrm{FWHM}=$ $0.986 \lambda)$. Using a blue laser diode $(\lambda=405 \mathrm{~nm})$, the FWHM equals $399 \mathrm{~nm}$ in radial polarization and $243 \mathrm{~nm}$ in circular polarization.

Fig. 5d and e depict the light confinements generated by circularly and radially polarized evanescent Bessel beams, respectively, when $\mathrm{NA}=1.3(n=1.5)$. Let us note that evanescent Bessel beams have been proposed to generate virtual tips for near-field microscopy [10]. From Fig. 5f, we see that in circular polarization, the spot (or virtual tip) lateral size $(\mathrm{FWHM}=0.5 \lambda)$ is 1.6 times larger than the one obtained in radial polarization $(\mathrm{FWHM}=0.32 \lambda)$. When $\lambda=405 \mathrm{~nm}$, the FWHM of $203 \mathrm{~nm}$ in circular polarization falls down to $130 \mathrm{~nm}$ in radial polarization. Therefore, depending on the chosen NA, a considerable gain of light confinement can be expected by choosing the right polarization state.

\section{Ultimate focal spots}

According to Fig. 4, coefficients $K_{1}^{\text {circ }}$ and $K_{1}^{\text {rad }}$ cancel for specific values of NA. We have $K_{1}^{\text {circ }}=0$ when NA $\ll 1$ whereas $K_{1}^{\mathrm{rad}}=0$ when $\mathrm{NA}=1$. In those particular cases, the enlargement of the light confinement by function $J_{1}^{2}$ vanishes. Therefore, the spot size is only limited by the central lobe of $J_{0}^{2}$ which leads to the ultimate focal spot in linear optical regime.

In paraxial regime, the spot size lowest limit is reached in circular polarization (and also in linear polarization). This case is well-known and can be described by scalar diffraction theory $[25,26,1]$. The physical explanation of spot size minimization in radial polarization when $\mathrm{NA}=1$ can 

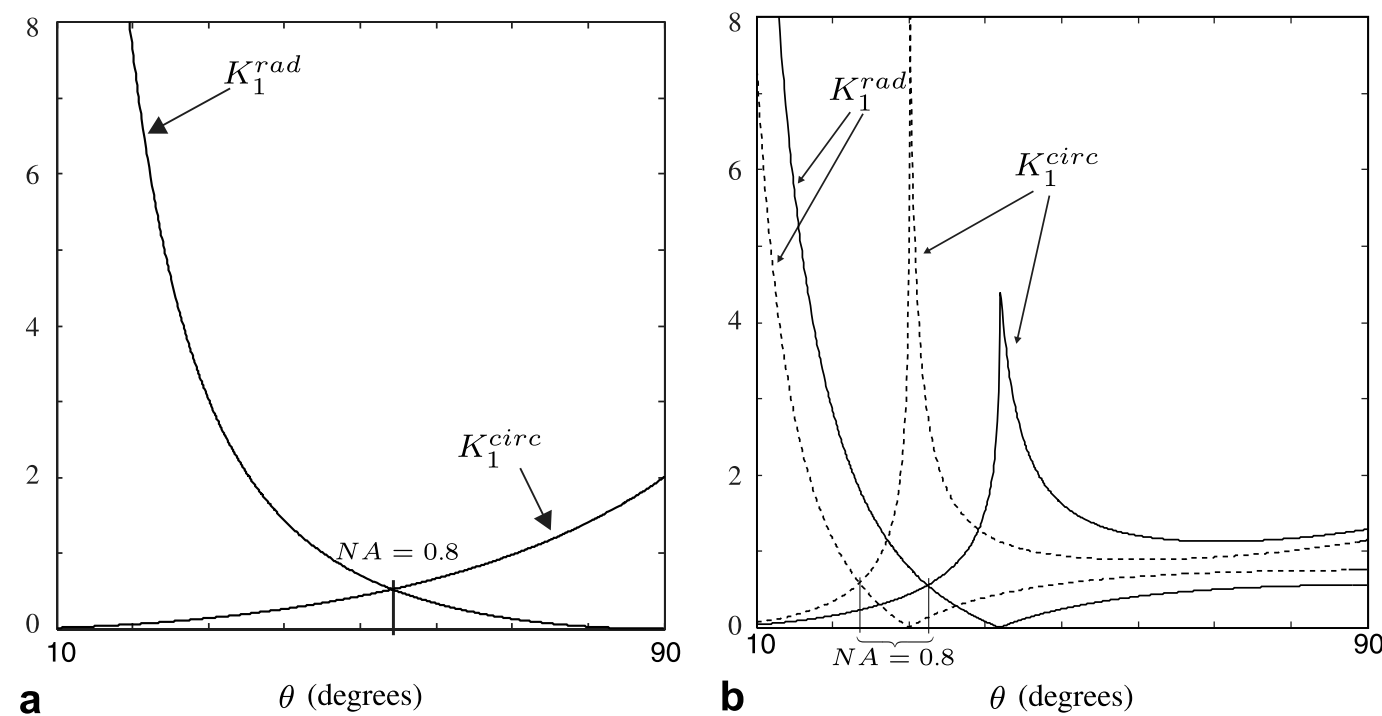

Fig. 4. Plots of coefficients $K_{1}^{\text {circ }}$ and $K_{1}^{\text {rad }}$ as functions of $\theta$. (a): $n=1$ (no immersion); (b): $n=1.5$ (solid curve) and $n=2$ (dashed curve).
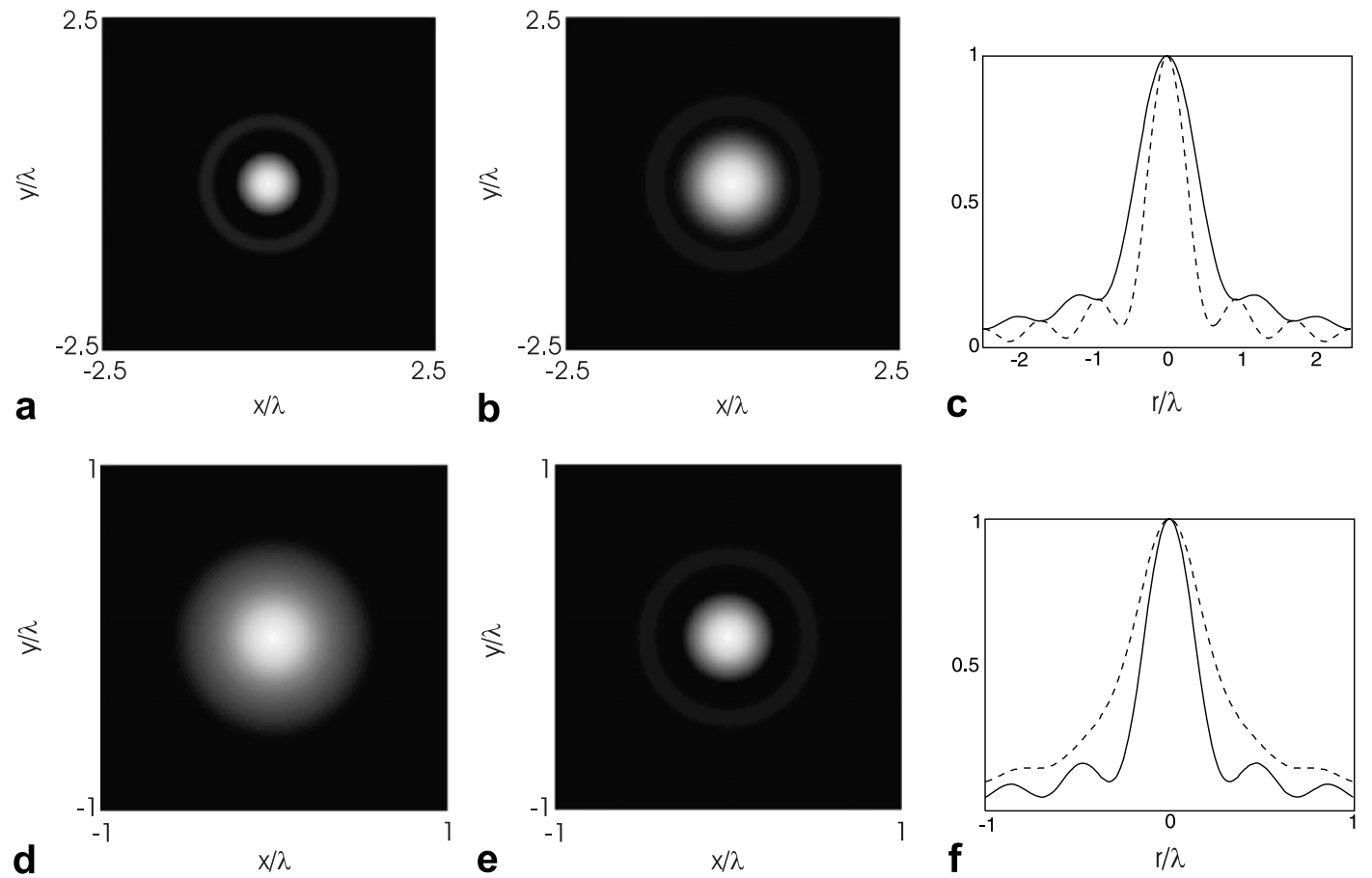

Fig. 5. (a)-(e): Bessel beam transverse intensities (simulations) when (a), (b) NA=0.65 and (d), (e) NA = 1.3, with (a), (d) circular and (b), (e) radial polarizations. (c) and (f): Intensity profiles in radial polarization (solid curves) and circular polarization (dashed curves).

be simply explained by means of the ray-tracing of Fig. 1 . In this case, the aperture angle $\left(\theta^{\prime}\right)$ of the transmitted Bessel beam is exactly $90^{\circ}$ from the normal $(0 z)$ of the interface. It turns out that the highly symmetric radial polarization makes each spectral component of the transmitted Bessel beams be p-polarized (proportional to $E_{\mathrm{p}}$ ). When $\theta=\theta_{c}$, all these components are oriented along the longitudinal direction $(0 z)$ : the transmitted Bessel beam is purely longitudinally polarized. Since the longitudinal field component of the radially polarized Bessel beams are proportional to $J_{0}$ [2], $I_{\mathrm{rad}}=\left|E_{z}\right|^{2} \propto J_{0}^{2}(\alpha r)$. Such remarkable properties have been approached numerically as well as experimentally by focusing light with spherical lenses combined to annular apertures $[27,28]$ or with parabolic mirrors [29]. Let us note that the ultimate spots, which are obtained with ideal Bessel beams, can be experimentally reached with enough accuracy when conical optics such as conical objectives [2] are considered.

The spot size lowest limit can be simply found as a function of NA and $\lambda$ by measuring the FWHM of function $J_{0}^{2}(\alpha r)$ and adapting the result to the well-known formula associated to Rayleigh criterion [26]. We obtain: 

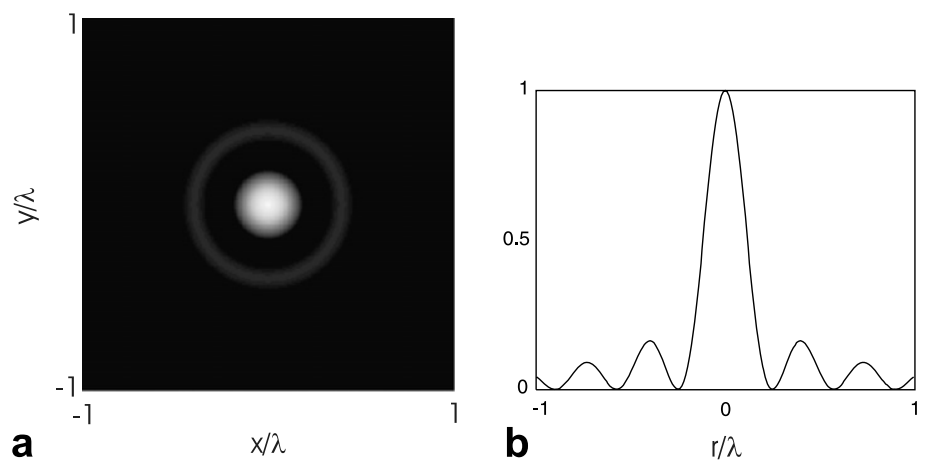

Fig. 6. Characterization of the ultimate focal spot generated at NA $=1.53$ (simulation). (a): Bessel beam intensity in $(x, y)$ plane; (b): Intensity profile (radial polarization).

$\mathrm{FWHM}=\frac{0.36 \lambda}{\mathrm{NA}}$

One of the most intriguing challenges for super-resolving microscopy is the achievement of the ultimate focal spots at the highest numerical aperture regime (NA $>1$ ). One possible way follows the analysis above-discussed. It turns out that the spot size lowest limit is reached in radial polarization when the index of refraction $n_{\mathrm{s}}$ of the medium containing the transmitted Bessel beam matches the numerical aperture (we have previously noted that the spot size minimization is observed when $\mathrm{NA}=n_{\mathrm{s}}=1$ ). In other terms, when $\mathrm{NA}=n_{\mathrm{s}}$, the propagative-to-evanescent regime limit of the transmitted Bessel beam is reached, leading to the cancellation coefficient $K_{1}^{\mathrm{rad}}$. This conclusion can be extended to the case NA $>1$ by generating Bessel beams in a medium whose optical index $\left(n_{\mathrm{s}}\right)$ is larger than 1 . This can be practically realized by immersing the transmitted evanescent Bessel beam in a high refraction index liquid, satisfying $n_{\mathrm{s}}=\mathrm{NA}$. In lithography and data storage applications, this can be also performed by adapting the index of the recording medium to NA [10]. Note that the technics based on conical lenses for generating Bessel beams are well-adapted for data storage since, unlike aperture-based systems, no energy is lost during the focusing process.

Fig. 6 displays the ultimate focal spot reached when $n=2$ (LASF35 Schott glass), $\theta=50^{\circ}$ and $n_{\mathrm{s}}=\mathrm{NA}=1.53$. Using blue laser diode $(\lambda=405 \mathrm{~nm})$, the spot size is limited to $95 \mathrm{~nm}$ (Eq. (17)). In a more technologically challenging case involving $n=3.3$ (GaP [30]), $\theta=45^{\circ}$ and $n_{\mathrm{s}}=$ $\mathrm{NA}=2.3$, the FWHM falls down to $65 \mathrm{~nm}$. Let us note that liquids of refractive index up to 2.3 are commercially available [31]. When optimized, optical focusing performances tend to be comparable to the ones currently encountered in local probe microscopy.

\section{Conclusion}

The influence of polarization on Bessel beam generation has been analyzed in order to find out the procedure which generates the smallest focal spots, whatever the NA of the focusing system. Focus maximal shrinkage in air requires two steps, namely the Bessel beam generation and the choice of circular polarization when $\mathrm{NA}<0.8$ and radial polarization when NA $>0.8$. A way has also been proposed to obtain the ultimate focal spot for $\mathrm{NA}>1$. It consists of choosing the right medium in which the light spot has to be generated. When the optical index $n_{\mathrm{s}}$ of the medium satisfy $n_{\mathrm{s}}=\mathrm{NA}$, propagative-to-evanescent regime is reached and the highly symmetric radial polarization induces the spot size minimization. The proposed solution makes the ultimate spots purely longitudinally polarized. When NA $>1$, the light confinement acts as a non diverging subwavelength stylus of light which can be applied as a near-field "virtual tip" for SNOM applications. Calculated optical confinements exhibiting FWHM up to $65 \mathrm{~nm}$ make virtual tip microscopy an interesting challenger to conventional local probe one, despite the fact that resolution is limited by the wavelength in the former case.

\section{References}

[1] R. Arimoto, C. Saloma, T. Tanaka, S. Kawata, Appl. Opt. 31 (31) (1992) 6653 .

[2] T. Grosjean, F.I. Baida, D. Courjon, Appl. Opt., in press.

[3] G. Toraldo di Francia, Nuovo Cimento (Suppl. 9) (1952) 426.

[4] Z.S. Hegedus, Opt. Acta 32 (7) (1985) 815.

[5] Z.S. Hegedus, J. Opt. Soc. Am. A 3 (1986) 1892.

[6] M. Martinez-Corral, P. Andres, C.J. Zapata-Rodriguez, M. Kowalczyk, Opt. Commun. 165 (1999) 267.

[7] M.A.A. Neil, R. Juskaitis, T. Wilson, Z.J. Laczik, Opt. Lett. 25 (4) (2000) 245

[8] I. Leiserson, S.G. Lipson, V. Sarafis, Opt. Lett. 25 (4) (2000) 209.

[9] C.-C. Sun, C.-K. Liu, Opt. Lett. 28 (2) (2003) 99.

[10] T. Grosjean, D. Courjon, J. Microsc. 202 (2001) 273.

[11] S. Quabis, R. Dorn, M. Eberler, O. Glöckl, G. Leuchs, Opt. Commun. 179 (2000) 1.

[12] S.M. Mansfield, G.S. Kino, Appl. Phys. Lett. 57 (24) (1990) 2615.

[13] T. Grosjean, D. Courjon, D. Van Labeke, J. Microsc. 210 (2003) 319.

[14] B. Richards, E. Wolf, Proc. Roy. Soc. London A (1959) 358.

[15] K.S. Youngworth, T.G. Brown, Opt. Express 7 (2000) 77.

[16] P. Török, P. Varga, Z. Laczik, G.R. Booker, J. Opt. Soc. Am. A 12 (2) (1995) 325

[17] D.P. Biss, T.G. Brown, Opt. Express 9 (10) (2001) 490

[18] L.E. Helseth, Opt. Commun. 191 (2001) 161. 
[19] S.R. Mishra, Opt. Commun. 85 (1991) 159.

[20] Z. Bouchal, M. Olivik, J. Mod. Opt. 42 (8) (1995) 1555.

[21] P. Vahimaa, V. Kettunen, M. Kuittinen, J. Turunen, A.T. Friberg, J. Opt. Soc. Am. A 14 (8) (1997) 1817.

[22] S. Ruschin, A. Leizer, J. Opt. Soc. Am. A 15 (5) (1998) 1139.

[23] L.E. Helseth, Opt. Commun. 257 (2006) 1.

[24] T.D. Milster, Opt. Photon. News (2005) 28.

[25] J. Durnin, J. Opt. Soc. Am. A 4 (4) (1987) 651.
[26] M. Born, E. Wolf, Principles of Optics, sixth ed., Pergamon Press, Oxford, 1980, Reprinted by Cambridge University Press, 1997.

[27] T. Wilson, F. Massoumian, R. Juskaitis, Opt. Eng. 42 (11) (2003) 3088.

[28] L.E. Helseth, Opt. Commun. 229 (2003) 85.

[29] N. Davison, N. Bokor, Opt. Lett. 29 (12) (2004) 1318.

[30] K. Hirota, T.D. Milster, K. Shimura, Y. Zhang, J.S. Jo, Jpn. J. Appl. Phys. 39 (1) (2000) 968.

[31] Cargille Labs. <www.cargille.com>. 